\title{
A Methodology for Investigation of Grain-Boundary Diffusion and Segregation
}

\author{
Zirong Peng ${ }^{1}$, Thorsten Meiners ${ }^{1}$, Baptiste Gault ${ }^{1}$, Christian Liebscher ${ }^{1}$, Dierk Raabe ${ }^{1}$ and Yifeng Lu ${ }^{2}$ \\ 1. Max-Planck-Institut für Eisenforschung GmbH, Max-Planck-Straße 1, Düsseldorf, Germany \\ 2. Institute for Informatics, Ludwig-Maximilians-Universität München, Oettingenstraße 67, München, \\ Germany
}

A grain boundary is the area where two grains of different crystal orientations join together. It is a type of two-dimensional crystal defects where lattice discontinuity and/or chemical inhomogeneity exist. Since the early 1980s, when Watanabe first introduced the idea of 'grain boundary design' [1], now often called grain boundary engineering (GBE), there has been an intensive effort to study grain boundaries. The key concept of GBE is by tailoring the structures of grain boundaries to improve the performance of polycrystalline and nanocrystalline materials. Scientists and engineers usually focus on the geometric characters of grain boundaries, which are defined by the orientation relationship between adjacent grains and the spatial orientation of the grain boundary plane. It was found that the properties and behaviors of grain boundaries largely depend on their geometric parameters [2]. For example, special grain boundaries with low- $\Sigma$ CSL (coincidence-site lattice [3]), i.e. $\Sigma 3, \Sigma 5, \Sigma 7$, etc. boundaries, have distinct properties such as lower energies, lower tendencies to trap impurities and solutes, lower electrical resistivity, as well as higher resistances to intergranular sliding or degradation as compared to random grain boundaries. Therefore, by tuning the grain boundary character distribution, desired bulk properties can be obtained. In recent years, with the development of advanced analytical techniques such as high-resolution (scanning) transmission electron microscopy (HR(S)TEM) and atom probe tomography (APT), grain boundary chemistry has drawn more and more attentions. Applications of those techniques have shown that grain boundaries can exhibit an array of different chemical states, i.e. clean or segregated with various elements, decorated with complexions or secondary phases, which resulted in significant differences in properties such as mobility, segregation and transport coefficients, mechanical, electric, magnetic, oxidation and corrosion behaviors. Accordingly, using compositional modifications to manipulate the properties of grain boundaries, and ultimately the performance of the material, has become a new concept for material design, termed as grain boundary segregation engineering [4] or complexion engineering [5]. In this context, understanding the formation of different grain boundary chemical states and identifying their compositions is of importance to the material science community.

In this work, we developed a simple, yet efficient, methodology: producing a compositional gradient along grain boundaries, which enables us to access various chemical states within a single investigation. We use a well-controlled diffusion annealing process to produce the compositional gradient. The specimen is a bilayer thin film sputter deposited onto a cemented tungsten carbide substrate (for more details about the sample, please see ref. [6]). One layer is the matrix, here in this study is nanocrystalline PtIr. The other layer is the diffusant, here Cr. In principle, this method can be applied to any material systems when proper conditions are chosen.

After annealing, a combination of various characterization techniques, including APT and (S)TEM, were applied. A dual beam scanning-electron microscope / focused ion beam (SEM/FIB) instrument (FEI Helios Nanolab 600/600i) was employed to prepare APT and TEM specimens from regions of interesting by a in-situ lift-out method. APT measurements were conducted using a Cameca LEAP 5000 XS device and (S)TEM analysis were done using a probe-corrected FEI Titan Themis 60-300 S/TEM operated at 
$300 \mathrm{kV}$. Figure 1 shows the results from such a complementary approach, as well as a composition profile obtained along a boundary by APT. To extract local compositional excesses at the boundary and the grain boundary diffusion coefficient, novel APT data analysis method has also been developed and will be discussed in this contribution. As readily visible from Figure 1, our work here demonstrates that a strong gradient of $\mathrm{Cr}$ can be generated at a grain boundary. This gradient of composition will affect the formation of e.g. grain boundary complexions. Therefore, such samples create the opportunity to investigate the grain boundary segregation and phase formation behavior as a function of the chemical potential.

\section{References:}

[1] T. Watanabe, Res Mech. Int. J. Struct. Mech. Mater. Sci. 11 (1984).

[2] F. Weinberg, Prog. Met. Phys. 8 (1959), p. 105.

[3] W. Bollmann, Crystal Defects and Crystalline Interfaces, (Springer, Berlin Heidelberg) (1970).

[4] D. Raabe et al, Curr. Opin. Solid State Mater. Sci. 18 (2014), p. 253.

[5] P.R. Cantwell et al, Acta Mater. 62 (2014), p. 1.

[6] Z. Peng et al, Corros. Sci. (2017) (Accepted).
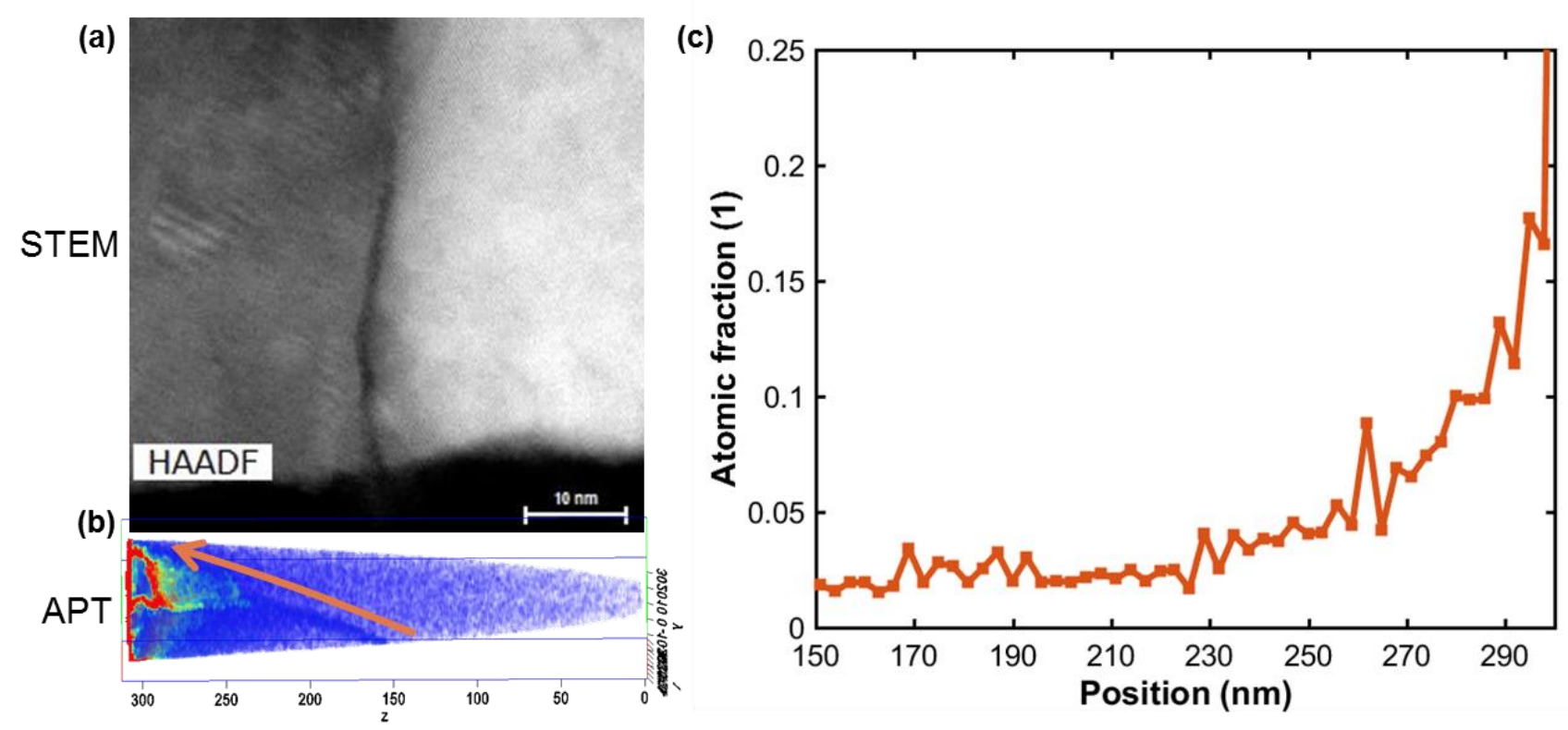

Figure 1. (a) HAADF-STEM image showing the diffusion and segregation of Cr along a PtIr grain boundary. (b) Blue-to-red rainbow concentration map of Cr. Blue indicates the lowest concentration and red indicates the highest concentration. (c) Amount of $\mathrm{Cr}$ at the grain boundary along the arrow marked in (b). 